\title{
El Deporte Inclusivo como Alternativa de Vida para los Jóvenes con Discapacidad
}

\author{
Inclusive Sports as an Alternative Life for Young People with Disabilities
}

\author{
Erica Tatiana Garavito-Campillo ${ }^{1}$ y Jesús Fernando-Kerguelen ${ }^{2}$
}

\section{EDICIÓN. Resiliencia}

Recibido: 22/febrero/2021

Aceptado: 10/junio/2021

P Publicado: 29/noviembre/2021

Páginas: 12-21

\&aís

${ }^{1}$ Colombia

${ }^{2}$ Colombia

\section{IIIII Institución}

${ }^{1}$ Facultad de Educación de la Universidad de Córdoba

${ }^{2}$ Facultad de Educación de la Universidad de Córdoba

\section{Correo Eletrónico}

1erikagaravitoc@correo.unicordoba.edu.co 2jesuskerguelens@correo.unicordoba.edu.co

\section{ORCID}

${ }^{1} \mathrm{https}: / /$ orcid.org/0000-0003-2324-0616 ${ }^{2} \mathrm{https}: / /$ orcid.org/0000-0002-7540-8102

\section{Citar así: Lf APA / IEEE}

Garavito-Campillo, E. \& FernandoKerguelen, J. (2021). El Deporte Inclusivo como Alternativa de Vida para los Jóvenes con Discapacidad. Revista TecnológicaEducativa Docentes 2.0, 1(1), 12-21. https://doi.org/10.37843/rted.v1i1.214

E. Garavito-Campillo y J. FernandoKerguelen, "El Deporte Inclusivo como Alternativa de Vida para los Jóvenes con Discapacidad", RTED, vol. 1, n. ${ }^{\circ}$ 1, pp. $12-$ 21, nov. 2021.

\section{Resumen}

A diario encontramos que cada persona tiene unas barreras, pero no límites. La discapacidad se hace evidente cuando se ponen barreras y estas a su vez restringen la participación de las personas en todos los contextos, cuando esta se presenta en los jóvenes esta pasa a un segundo plano debido a que estos son más fuertes, hacen de ella una aliada. En la presente investigación se reflexionó sobre las limitaciones físicas que han tenido algunos jóvenes. El estudio evidencio los procesos inclusivos que actualmente se están dando en el ámbito del deporte adaptado, resaltando las diferentes historias de vida de jóvenes con discapacidad. Se realizó una búsqueda de documentos y trabajos que giran en torno al término de la inclusión, lo cual, genero una investigación cualitativa que tuvo como objetivo analizar cómo el deporte inclusivo fortalece las historias de vida de jóvenes con discapacidad en el departamento de Córdoba. La población estuvo representada por 14 jóvenes con discapacidad intelectual, auditiva y física, se eligió una muestra de 7 jóvenes que practican deporte adaptado. Luego, se realizó la observación directa y entrevista semiestructurada para la elaboración de los relatos de historias de vida. Por último, el análisis nos llegó a comprender que la práctica del deporte adaptado contribuye a mejorar la calidad de vida dándole sentido a lo que hacen, fortaleciéndose cada vez más como persona.

Palabras clave: Deporte inclusivo, discapacidad, historias de vida, alternativa de vida.

\section{Abstract}

Every day we find that each person has barriers, but not limits. Disability becomes evident when obstacles are put in place. These, in turn, restrict the participation of people in all contexts; when it occurs in young people, it goes to the background because they are more robust; they make it an ally. In this research, we reflected on the physical limitations that some young people have had. A search was carried out for documents and worked around the term of inclusion, which generated qualitative research that aimed to analyze how inclusive sport strengthens the life stories of young people with disabilities in the department of Córdoba. The population was represented by 14 young people with intellectual, hearing, and physical disabilities, a sample of 7 young people who practice adapted sports was chosen. Then, carried out direct observation and a semi-structured interview to elaborate on the stories of life stories. The study evidenced the inclusive processes currently taking place in adapted sport, highlighting the different life stories of young people with disabilities. Finally, the analysis understood that the practice of adapted sports contributes to improving the quality of life by giving meaning to what they do, becoming increasingly more robust as a person.

Keywords: Inclusive sport, disability, life stories, alternative life. 


\section{Introducción}

A diario encontramos que cada persona tiene unas barreras, pero no límites. La discapacidad se hace evidente cuando se ponen barreras y estas a su vez restringen la participación de las personas en todos los contextos, cuando esta se presenta en los jóvenes esta pasa a un segundo plano debido a que estos son más fuertes, hacen de ella una aliada. En el departamento de córdoba hay muchos jóvenes discapacitados que han demostrado que con la ayuda del deporte no tienen límites y crean sus propios sueños.

En el departamento de Córdoba según cifras de la Liga deportiva de discapacidad física existen alrededor de 220 Jóvenes con discapacidad quienes pertenecen a los procesos deportivos departamental, para este estudió se clasificaron 14 historias de vida, a diferencia de otras personas con discapacidad estos jóvenes gracias al deporte han encontrado un camino de esperanza, estos no se han enfocado en su condición médica, se han llenado de valor usando su discapacidad como un trampolín para alcanzar sus sueños, estos guerreros decidieron enfocarse en el deporte adaptado, deportes como Paracycling futbol7pc, boccia, para atletismo, concentrando todas sus energías en ellos, resaltando que esto los llena de vida, y los motiva a seguir adelante cada día.

En la presente investigación se reflexionó sobre las limitaciones que han tenido algunos jóvenes debido a que presentan unas limitaciones físicas que los han llevado a cambiar su forma de actuar y pensar ante la vida, y esto se debe a que presentan una discapacidad, la cual es entendida por la (OMS, 2001), como las deficiencias, limitaciones de la actividad y restricciones de participación. Las deficiencias son problemas que afectan a una estructura o función corporal; las limitaciones de la actividad son dificultades para ejecutar acciones o tareas. Por ello, el autor MontesDelgado et al. sustenta que "el deporte adaptado favorece el desarrollo personal y social del individuo que lo practica ya que facilita la superación y la inclusión a los entornos sociales" (2016, p.1).
En este artículo se buscó reflejar las historias de vida de estos jóvenes los cuales han mostrado un dinamismo, un cumplimento en cada una de las metas que se han propuesto, llevando un gran mensaje donde no existen limitaciones, físicas ni mentales, solo actitudinales en cada ser. Con esto se buscó analizar cómo el deporte inclusivo fortalece las historias de vida de jóvenes con discapacidad en el departamento de Córdoba.

\section{Metodología}

Para dar sustento a la investigación se realizó una búsqueda de documentos y trabajos que giran en torno al término de la inclusión, por lo que se cita una parte de las referencias investigadas sobre la problemática. Por lo tanto, la presente investigación se fundamentó bajo el paradigma cualitativo que de acuerdo los autores Blasco-Mira \& Pérez-Turpin señalan que la investigación cualitativa estudia la realidad en su contexto natural y cómo sucede, sacando e interpretando fenómenos de acuerdo con las personas implicadas (2007, p.25). Además, Hernandez-Sampierí et al. "sustenta que la investigación cualitativa también se lleva a cabo una intensiva revisión de la literatura" (2014, p.365).

Para atribuir el sentido de las historias de vida de los entrevistados se analizó las capacidades de comunicación con relación al contexto social tal como lo señala el autor Alheit "resulta posible pensar en la formación como trabajo individual de gestión de identidad" (2008, p. 41). Lo cual hace parte de la investigación cualitativa, por eso se aborda este estudio desde este paradigma, donde la realidad es construida socialmente mediante definiciones individuales de una determinada situación (Taylor, 2016) se parte de la información obtenidas de la realidad que viven estos jóvenes, se construyen sus relatos.

La población sujeta de estudio estuvo representada por 14 jóvenes con discapacidad con ganas de salir adelante y el deporte inclusivo es una de sus motivaciones, la muestra se seleccionó mediante el criterio de la intencionalidad en ese sentido se procedió 
a escoger a 7 jóvenes con discapacidad intelectual, auditiva, física, que practican deporte adaptado.

Las estrategias de recolección de información que se llevaron a cabo están determinadas por el tipo de investigación (cualitativa) se realizó la observación directa y la entrevista semiestructurada para la elaboración de los relatos de historias de vida. Para ellos se ha convertido en fortaleza en sus prácticas deportivas, es por esta razón que esta investigación es de gran impacto en el departamento de Córdoba.

Tal es el caso del estudio realizado por Salazar \& Escós (2020) se dedica a conocer la importancia que tiene la inclusión en la educación y también en el mundo del deporte, con lo que se va a dar a conocer las principales características de lo que es inclusión, y de cómo puede ser su incidencia en el proceso de enseñanza aprendizaje para los diferentes alumnos de los colegios. Así mismo, se propone una intervención práctica dirigida a la etapa de primaria. Para completar esta propuesta se realizaron 3 cuestionarios que estarán dirigidos a 3 públicos diferentes relacionados con la educación, como son los profesores, las familias y los alumnos, y de esta manera poder conocer cómo se trabaja este tema en los colegios y como se transmite tanto a alumnos como a sus familias. Esta investigación es de relevancia porque resalta la importancia que tiene la inclusión en el deporte.

Ahora bien, es importante referenciar el trabajo de Gómez (2019) el cual propone retomar historias de vida de profesores de Educación Física con discapacidad, con relatos que emergen con la decisión de ser profesor, junto a las eventualidades e incongruencias vividas en la formación inicial, comienzo de su ejercicio, idealización docente y posición frente a la formación continua, los cuales constituyen los ejes sobre los que giran estas historias de vida, como una oportunidad hacía una acción y compromiso pedagógico. Se realizó a 3 docentes quienes cuentan sus historias de vida desde la labor docente, y llega a la conclusión de que todas las metas se pueden superar siempre y cuando se le ponga el interés necesario y el empeño para salir adelante, integrar a aquellas personas con alguna dificultad ya sea cognitiva o física es dar el primer paso para generar equidad en la sociedad, y reconocimiento de las inmensas posibilidades de una persona con discapacidad.

En última instancia, se referencia un proyecto sobre la tesis de inclusión laboral de docentes con discapacidad visual en el campo educativo, elaborado por Gómez-Rodríguez (2017) el cual establece identificar en el escenario del ejercicio profesional del docente en condición de discapacidad visual, las políticas, culturas y prácticas; que en materia de inclusión laboral dificultan o favorecen su trabajo en la escuela. Este proyecto se planteó que todas las personas sin ningún tipo de discriminación pueden acceder, en igualdad de oportunidades y garantías; a la formación y trabajo; como, además, demostrar que se están implementando acciones que garantizan la participación dentro de un enfoque diferencial, universal y de derecho, las cuales, están eliminando las barreras físicas $\mathrm{y}$ culturales.

Ahora bien la siguiente investigación por Díaz-Better \& Eusse-López (2021) exponen como la educación física y sus maestros, a nivel mundial, afrontan a diario desafíos significativos relacionados en especial con el desarrollo de sus temáticas en el aula de clases, el comportamiento de los estudiantes, el material didáctico, la infraestructura, entre otros; debido a lo anterior se plantea la pregunta, ¿qué tanto se está dedicando a rediseñar el maestro de educación física cuando en sus escuelas se encuentran matriculados estudiantes con alguna discapacidad física? El objetivo principal analizar estudios que usan el deporte Boccia como herramienta de inclusión el aula de clases de educación física, para determinar cómo los deportes adaptados direccionan a la participación de todos los estudiantes en esta área del conocimiento.

En el método se realizó un mapeo sistemático de literatura en las bases de datos Science Direct, Springer Link, Taylor \& Francis, Scopus y JSTOR. Resultados: se recuperaron 718 documentos, de los cuales se identificaron $10(1,39 \%)$ trabajos relevantes 
para esta investigación. Conclusión: este mapeo muestra que hay un vacío de conocimiento con respecto al uso del deporte Boccia en las clases de educación física $y$, más aún, siendo un deporte paralímpico que pueda dar nuevas bases para la inclusión en las aulas de clases.

En un quinto estudio realizado por Pérez (2013) analizaron el grado de implantación del ciclismo a nivel internacional y los indicadores concretos que lo caracterizan. El estudio se llevó a cabo en el Campeonato del Mundo de Paraciclismo en Canadá en 2010. Se administró un cuestionario ad hoc de 20 preguntas a un representante cualificado de cada expedición evaluando los ámbitos de gestión, necesidades, aspectos clave y opinión sobre los procesos de integración del ciclismo en su país. Los autores concluyeron que las discapacidades menos integradas fueron la auditiva y la intelectual mientras que los aspectos más valorados en los procesos fueron la determinación de normativas específicas, la formación de técnicos deportivos, la existencia de una institución garante de los procesos y el apoyo económico específico.

\section{Resultados}

Una vez aplicados los instrumentos se procedió con el análisis de los resultados, haciendo uso de la triangulación de información, se contrasta la teoría, lo observado, los datos que arrojaron las entrevistas. En este estudio se emplearon entrevistas semiestructuradas permitiendo recoger la información necesaria de las historias de vida de los participantes quienes estuvieron motivados a contar su relato. Cuando se narra una vida no sólo se recoge lo que se ha aprendido en ella, sino que en el acto de narración mismo acontece un aprendizaje.

El proceso inicia con la narración de él entrevistado número uno (1), quien nació en Montería, Córdoba, el 27 de junio de 1994 más conocido como "El medallista de oro", es un atleta Cordobés, de 23 años, quien tiene una enfermedad llamada Ataxia de Friedreich es una afección neurológica hereditaria poco frecuente, que se presenta por primera vez entre los cinco y 15 años de edad. Inicialmente provoca torpeza en los movimientos y progresa a la inestabilidad en la posición de pie y al caminar, con dependencia de la silla de ruedas en la adolescencia tardía o al principio de los veinte años. Habitualmente, el habla se vuelve difícil. Un gen defectuoso específico se debe heredar de cada padre para que la enfermedad se desarrolle en el hijo (herencia recesiva autosómica). El entrevistado presentó todos los síntomas descritos (Kearney, Orrell, Fahey, Brassington \& Pandolfo, 2016)

Es un joven que nació muy saludable, pero la enfermedad apareció casi al culminar su educación básica secundaria, se percató de esto cuando se dio cuenta que sus movimientos se dificultaban, se caía sin razón alguna, de inmediato sus padres sospecharon porque su enfermedad es congénita, su hermano mayor también la padece, sin embargo, él es un gran luchador.

Un día determinó que esta enfermedad que poco a poco se apodera de sus movimientos no iba ser impedimento para convertirse en un hombre exitoso. El cual ha sido motivo de orgullo para toda su familia, además ha sido inspiración para su hermano quien está en condiciones similares debido al mismo diagnóstico. Las ganas de hacer cosas por su vida lo han llevado a ganar medallas, viajar por el mundo. Este joven practica para atletismo, ha ganado varias medallas, entre esas 5 de bronce, 1 de plata (a los 16 años de edad, la ataxia de Friedrich apareció en su vida). Desde entonces para movilizarse, utiliza una silla de ruedas, es un joven apasionado, lo motivan las ganas de salir adelante, aunque, veces se deprime porque cuando habla se le entiende poco y le cuesta respirar.

Este chico, vive en un barrio al sur de Montería con su esposa, ella lo atiende con amor, le brinda el calor de hogar, lo ayuda a trasladar a donde quiera, ella es su motivación más grande, quien lo ayudó a montar su primer negocio, es una tienda de víveres $\mathrm{y}$ abarrotes, expresa a sus 23 años ha sido difícil no sentirse como cualquier joven, pero está feliz, orgulloso de lo que es y lo que tiene. 
Sus padres viven cerca lo visitan periódicamente, su madre siempre le da un consejo y una palabra de aliento, se siente feliz de traer medallas y victorias a su amada tierra (Montería), ser reconocido en la localidad y recibir elogios en las calles lo llena de satisfacción. Además, expresa que nunca ha vivido momentos donde se sienta discriminado, considera que siempre se ha encontrado con la cara amable de la sociedad, quiere seguir trabajando y entrenando para llegar a ser el gran medallista de oro, ser reconocido mundialmente, para que todo los Colombianos un día se sientan orgullosos en su representación, demostrarle a la sociedad que la voluntad y las ganas de salir adelante son más grandes que cualquier enfermedad. (RE-1, J2, L121-140). Esta superación de la discapacidad depende de cada persona, es decir como lo asimila, lo afronta y lo vuelve su realidad.

En el estudio se resalta una segunda historia de vida del entrevistado dos (2) un joven de 17 años de edad nació en la ciudad de Montería Córdoba. Quien presenta una discapacidad congénita llamada parálisis espástica, la cual compromete sus piernas, la resistencia. Durante sus primeros 9 años de vida él no tuvo inconvenientes con esta discapacidad, pero, cuando cumple los 10 años empezó a sentir una debilidad en sus piernas, pero esto no fue un impedimento para que nuestro querido amigo gozara plenamente de su infancia. Hasta ese momento él podía disfrutar libremente del juego pero poco tiempo después su discapacidad se hacía más notable sobre todo cuando practicaba su deporte favorito, el futbol, pues como mencionamos antes esta discapacidad compromete sus piernas afectando su parte física, se le dificulta correr largas distancias o por tiempos prolongados.

Generalmente cuando una persona tiene una discapacidad se le considera vulnerable, además es discriminada por muchos. En su caso no ha tenido que pasar por una situación de estas, o por lo menos él no ha se ha sentido discriminado fuertemente por alguien, en su defecto si llegase a suceder su mente, corazón, se sienten preparados pues él ha aprendido a amar su condición. Desde que es consciente de su discapacidad no se ha sentido excluido en ninguna etapa de su vida, pues ha recibido total apoyo de su familia, vecinos, amigos.

Además, gradece tener su discapacidad gracias a ella ha logrado muchos sueños. Su apoyo más grande emprendió con la selección Córdoba, esta le ha permitido sentirse orgulloso de sí mismo, valorar la vida aun con todas las dificultades del mundo. Lo ha motivado a seguir adelante, a ver su discapacidad como una oportunidad que asegura no haber tenido a no ser por su condición. Gracias a ella ha tenido la dicha de competir en torneos en la ciudad de Medellín y uno de los momentos más felices de su vida fue viajar y jugar en esa ciudad, darse cuenta de que él no es el único, también otros que luchan, personas sonrientes, siendo felices, aprovechando esta corta pero significante vida.

El entrevistado 2 sonríe, se siente vivo cada vez que pisa una cancha para jugar futbol se siente orgulloso de obtener una medalla para su selección. Para él aún siguen los sueños. Deseaba ingresar a la Universidad, estudiar licenciatura en educación y reporte, debido a su esfuerzo logra una beca, otras de sus metas a mediano plazo es lograr un cupo en la selección de futbol $7 \mathrm{pc}$ de esta forma representar a su país con orgullo (RE, J3, L180.198). En algún momento sintió angustia, frustración cuando este fue rechazado para la selección, expresa que ha sido uno de los momentos más tristes, pero no está solo, tiene claro que rendirse jamás es una opción, considera importante superar cualquier obstáculo, para cumplir sus sueños.

Hoy nos invita a todos a perseguir "los sueños por más piedras que se atraviesen, la discapacidad abre puertas no pensemos que las cierra, no hay límites, se puede ser feliz, no te rindas" estas son sus palabras de ánimo. Tiene muy claro que soñar es vivir; dentro de 10 años él se ve siendo un profesional, cumpliendo sus metas, alcanzando sus planes. Actualmente sigue practicando el deporte de futbol $7 \mathrm{pc}$ el cual significa todo para él, pues le ha mostrado lo valioso que somos cada uno de nosotros, además, ha conocido personas maravillosas y amistades valiosas, sin ninguna duda su deporte es lo mejor que le ha 
pasado. Con esta historia se tiene presente que, todo final anuncia un nuevo comienzo.

Estas dos historias de vida que se han referenciado refleja como la aceptación social es tan importante uno de los objetivos de cualquier programa deportivo debe consistir en desarrollar conductas socialmente deseables utilizando adecuadamente el autocontrol, En general, los beneficios de la práctica de actividades físicas y deportivas para las personas con discapacidad son básicamente los mismos que cualquier persona, Arraez (2013) afirma que al practicar actividad física las personas con discapacidad mejorarán su eficiencia física y fisiológica, reducirán su estrés y ansiedad, se sentirán intelectualmente estimuladas.

Ahora bien, Muchas veces nos dejamos intimar por los retos que la vida nos trae, en ocasiones renunciamos a nuestros sueños y nos dejamos vencer por los diferentes obstáculos que se nos presentan. Esta es la historia del entrevistador 3 un joven soñador oriundo de la ciudad de Montería - Córdoba, nacido el 14 de agosto de 2001 actualmente 19 años. Es un chico alegre, espontáneo vive en el barrio Mocari de esta localidad. Es el primer hijo de 2 hermanos. Desde su nacimiento ha estado presente un trastorno llamado parálisis cerebral lo que dificulta sus movimientos, tonalidades en su voz. Este joven se ha enfrentado desde muy niño a grandes desafíos, como las constantes terapias físicas que se realizaron desde muy pequeño durante un lapso de 10 años de vida, caminó por primera vez cuando tenía 3 años de edad. (RE, J3, L203-228). Sus padres han sido los pilares fundamentales lo apoyan, le han enseñado a ser un joven independiente. Lo que hace a este un chicho luchador, con ganas de salir adelante.

Muchas personas apoyan, mientras que otras personas con falta de conocimiento acerca de la situación lo intimidan, cuando entro a la escuela primaria algunos de sus compañeros se reían de él, pero este no se dejó "los bobos son ustedes, que no han viajado nunca" esta es la respuesta sujeta de estudio número 3 , daba a las personas con falta de empatía. Cabe resaltar, el deporte ha hecho de él una persona más sociable, empática, feliz.
Inicio en el deporte a muy temprana edad, primero práctico atletismo, actualmente práctica futbol 7 el cual es un deporte adaptado, comenzó a jugar futbol porque siempre le ha llamado la atención, pero luego gracias a un buen amigo y entrenador de este deporte me motivó a practicarlo ( $\mathrm{RE}, \mathrm{J} 3$, L226-234).

"Yo soy feliz, he viajado por muchas partes, he ganado muchas medallas" resalta el joven, el deporte lo hecho una persona fuerte, que ya no se deja intimidar por los obstáculos. A futuro se ve como profesor en educación física, dice seguir practicando el fútbol porque esto lo apasiona. Para finalizar el joven deja un mensaje a las personas que dicen no puedo "sigan adelante". Este relato refleja la importancia que cumple la motivación en la vida de las personas, contrastando esta situación con la teoría de Bandura (1986) la cual establece un aspecto importante de la conducta humana: que las personas crean y desarrollan sus autopercepciones acerca de su capacidad, mismas que se convierten en los medios por los cuales siguen sus metas, y controlan lo que ellos son capaces de hacer para controlar, a su vez, su propio ambiente.

Debido a esto se hace mención la historia de vida de Entrevistado 4, es un joven de 15 años, nacido de San Antero, el cual en algunos años podría convertirse en uno de los mejores campeones de las pistas, es posible que tenga una oportunidad de mostrárselo al mundo. Antes de apresurarnos al futuro queremos contarte un poco acerca de cómo ha sido su infancia.

Este joven tiene diversas capacidades, pero también tiene una discapacidad física, la cual ha convivido siempre con él, desde su nacimiento. Pues, le falta la muñeca de su brazo, su familia ha estado a su lado siempre apoyándole, haciéndole ver que es capaz de mucho. Este joven cuenta, nunca ha sido discriminado, cabe resaltar que siempre las personas lo quedaban observando, pero nos dice que nunca se sintió incomodo que siempre se sintió bien tal y como Dios lo envió al mundo, es un niño con una autoestima de admirar, un joven que tiene una confianza y seguridad en sí mismo, que incluso una persona "normal" muchas veces 
no la tiene, escucharlo hablar con esa seguridad y ese entusiasmo contagia su alegría y felicidad a los demás, por lo menos así lo sentimos.

Quien relata en la entrevista que cuando era niño se le dificultaba manipular cosas con las manos, en especial amarrarse los cordones para él fue un desafío, el cual cabe resaltar que no fue grande para él, al pasar el tiempo pudo aprender y lograr amarrase los cordones (RE, J4 L246-257).

$\mathrm{Su}$ motricidad gruesa está completamente bien es capaz de correr, saltar, bailar, caminar, hacer diversos ejercicios con su cuerpo, pero no se puede decir lo mismo de su motricidad fina, esto debido a su mano derecha se le dificulta un poco el poder manipular las cosas, como por ejemplo amarrarse los cordones, vestirse, comer, entre otras cosas. Para él fue un gran desafío aprender a escribir, comer, atrapar, entre otros. Nunca pierde las ganas de salir adelante, demostrarles a todos que si se puede, se vale soñar en grande, sin importar la discapacidad o problemas tengas, siempre debes luchar por tus metas, tus sueños, nunca rendirse.

Escuchar hablar a este joven con tanta motivación, alegría, certeza, contagia a cada persona de luchar por sus sueños, expresa tener ganas de comerse al mundo, salir adelante, cumplir sus metas. Nunca imaginó estar dentro del deporte, tampoco que llegaría tan lejos, aunque se sentía un joven igual, con las mismas capacidades que cualquier otro. Un día llego un amigo, lo invito a entrar al grupo de atletismo, con el entusiasmo que lo caracteriza dijo si, Comenzó su vida como atleta, observó al pasar los días, que cada vez le gustaba entrenar, participar más en todas las actividades, desde ese momento supo que continuaría en ese camino y siempre estaría con la mejor actitud.

Al principio fue un poco frustrante para él, teniendo en cuenta que le tocaba competir con niños más grandes, quienes siempre le llevaban un poco de ventaja, esta situación lo hacía desestabilizarse emocionalmente, sin embargo, nunca se rindió, siempre decidido a dar lo mejor de sí, tanto que expresa los mejores momentos para él fue cuando gano un campeonato: donde tuvo la oportunidad de ganar 2 medallas de oro, una de plata. En ese día estableció tres récords, fue algo asombroso para él. Afirma que su mayor habilidad es la resistencia porque cuando va llegando a la meta y observa que se lo van pasando no permite que lo hagan, resiste hasta que puede ganar sin bajar la guardia.

Ahora bien, hablemos de su motivación, lleno de juventud cuenta que su motor para seguir adelante es su familia, está siempre ha permanecido junto a él apoyándolo, dándole amor y consejos, nunca lo han dejado solo en este duro camino, comenta que su deseo más grande, es darle una casa a su madre. Su meta actual es ser el mejor atleta paralímpico del mundo, actualmente tiene 15 años y sus sueños llegan a la luna. Citando a Cajamarca (1994) dice que la motivación es el impulso interno que induce al educando a decidir construir su aprendizaje, es hacer con entusiasmo lo que se está haciendo; aunque el ambiente, y la familia crean las condiciones ayudan a la motivación, esto es lo que tiene este joven un impulso interno tal como lo expresa el autor ese motor que lo mueve para su caso sería ser el mejor atleta.

Con tan pocos años de edad, escucharlo hablar con tanta pasión, con tantas ganas de salir adelante, con una discapacidad, que es su fortaleza, cumplirá el propósito por el cual Dios lo mandó a este mundo, después de todo cuenta se ve en el futuro siendo un gran deportista profesional. "En realidad, no existen personas discapacitadas sólo personas con distintos grados de aptitud" (RE, J4 L260264).

Ahora la siguiente historia de vida entrevistado cinco (5). Es un joven que desde niño se ha caracterizado por su carisma, alegría y contagio que despierta al hablar, nació en Sahagún Córdoba en una familia conformada por sus padres, dos hermanas siendo gemelo de una de ellas, vivé con sus padres. Los primeros 8 años los vivió en Magangué, tuvo una infancia tranquila, a los 5 años comenzó a practicar BMX callejero donde poco después se convirtió en su pasión. Hace 4 años se mudó al municipio de Sahagún, por conflictos externos en su barrio, el cambio le proporcionó la oportunidad de 
estar un poco más tranquilos, y con mejores oportunidades para el deporte (RE, J7, L290307).

A su corta edad le tocó enfrentarse a un cáncer desarrollado por un hematoma producido por un accidente en una pista de BMX el cual le costó su pierna derecha. Al enfrentarse con esta difícil situación el entrevistado 5 solo pensaba en que sus metas trazadas en este deporte se esfumarían y no volvería a montar bicicleta, pues faltarle una pierna y contar con pocos recursos para una prótesis, debido a sus escasos recursos era lo que pasaba por su mente. Estos y muchos interrogantes más aparecieron, eran tantas preguntas pero la respuesta era una sola su motivación, esperanza de no darse por vencido eran lo único que tenía a su favor, después de largas jornadas de quimioterapia, la amputación de su pierna renació como el ave fénix, con muchas ganas de volver a las pistas.

En sus años escolares hubo momentos de discriminación hacia su aspecto físico, pero lo tomo de la mejor manera para que esto no se convirtiera en un impedimento. Cuenta que diario veía a un amigo el cual vendía comida en una bicicleta para llevar el pan a su mesa, pero lo que lo hacía especial era que se encontraba en las mismas condiciones sin una pierna, ver a diario ese ejemplo me convencía cada vez más que se puede creer en grande. (RE, J5, L310-325); Empezó a ir a municipios como la Ye y Colomboy donde se encontraba con ciclistas que poco después lo acompañaban en sus recorridos. Un día llega a su vida un profesor quien le adapta su bicicleta y a través de fundaciones recibe artículos deportivos que lo animaron a emprender su meta de ser un gran ciclista a pesar de su discapacidad.

Actualmente tiene 19 años, es jefe de la manada los Boys scout es integrante de la selección Córdoba de Paracycling, y su mayor sueño es ser entrenador de paracycling en el departamento de Córdoba. También se dedica a dictar charlas motivacionales $y$ se ha convertido en un ejemplo para muchos; todas las personas que lo conocen o han tratado con el concuerdan en que es un excelente ser humano, que esta echo para grandes cosas y que sin duda alguna ha demostrado ser un campeón. La frase "los sueños andan en bicicleta" ha marcado significativamente la vida de nuestro campeón, todos aquel que tiene el placer de conocerle se contagian al ver su carisma.

Sujeto de estudio número seis (6), este joven su vida tuvo un giro a través del deporte. Quien actualmente tiene 21 años, desde su nacimiento, le detectaron parálisis cerebral, afectando así sus dos pies, y la mano derecha, estudia en la universidad de Córdoba, quería estudiar ingeniería en sistemas, pero no se sentía seguro de ejercerla, sin embargo, su familia lo apoyo y se decidió a estudiar licenciatura en informática, con el fin de ser docente y llevar un gran mensaje a los jóvenes.

En su infancia poco asistía a la escuela, ya que por su discapacidad tenía que pasar más días en una clínica, esto ocasionaba que se relacionara poco con otros niños, y vivir a plenitud, a medida que pasaba el tiempo y tomaba conciencia de su condición, que adaptarse no sería una tarea fácil pero decide emprender, termina sus estudios académicos, alternados con el deporte, desde el año 2012 practica futbol $7 \mathrm{pc}$ el cual le ha permitido participar en algunos campeonatos, viajar a otros países llevando el nombre de Colombia muy alto, obtuvo el tercer puesto en Sao Pablo- Brasil una de sus mejores experiencias y momentos más felices de su vida ya que tenía claro que el deporte le cambiaría la vida pero no esperaba que fuera tanto (RE, J6, L370). Es fuente de motivación para otros jóvenes en el departamento de Córdoba gracias a sus logros adquiridos.

La última historia de vida por contar en este artículo es la de la entrevistada número siete (7), es una joven de 24 años, quien tiene un diagnóstico Atrofia Muscular espinal (AME) es una enfermedad neuromuscular, de carácter genético, se manifiesta por una pérdida progresiva de la fuerza muscular. (Esto ocurre debido a la afectación de las neuronas motoras de la médula espinal, el impulso nervioso no se pueda transmitir correctamente a los músculos produciendo que éstos se atrofien.) Ella cuenta: que fue un bebé muy sano, sin embargo, al cabo de un 
año y medio empezó a perder las fuerzas en sus piernas, ya no podía caminar, tener equilibrio, coordinación.

Cumplidos los tres años de edad dejó de caminar y es allí cuando le diagnosticaron esta discapacidad, ella cuenta que su infancia fue como la vida de cualquier otra persona, convivió y se relacionó con todos los que estaban a su alrededor, gracias a su forma de ser tan extrovertida, espontanea, sin embargo en la adolescencia tuvo en los que se sentía excluida por parte de otras personas, especialmente en el comienzo de su adolescencia, relata que en grado octavo de bachillerato a su grupo le fue asignado un aula en un segundo piso sin tener en cuenta su condición física ya que se transporta en una silla de ruedas, cuando se solicita e cambio muchos de sus compañeros estaban en contra, pues decían que por una sola persona no era justo el cambio de aula, ese episodio la marcó mucho (RE, J7, L405-414).

Sin embargo, considera que se siente una persona afortunada cuando juega boccia, el cual es uno de los deportes paralímpicos más populares, ella explicó que "cada jugador o pareja de boccia tiene 6 bolas de un color (rojas o azules) llamadas boccias. La partida comienza cuando se lanza una bola blanca, que queda parada en el centro del campo de juego, en donde el jugador debe lanzar sus boccias con el objetivo de acercarse lo máximo posible a la bola blanca. Quien lo consiga con mayor acierto, gana". Este deporte le cambió mucho la vida, además sueña con quedar de campeona. Este deporte le ha brindado la oportunidad de conocer a otras personas con diferentes discapacidades, darse cuenta de que son capaces de hacer lo que se propongan y que la limitación solo está en la forma de pensar que se tenga, hasta la fecha no ha ganado medallas como otros pero en su departamento es de las pocas que practica este deporte (RE, J7, L480-492).

\section{Conclusiones}

En la actualidad se ha visto reflejado que la inclusión y discapacidad son temas que se han tomado mucha fuerza, lo que ha permitido que las personas que presentan alguna discapacidad tengan mejores oportunidades, el deporte ha sido uno de los puentes que más ha contribuido a que en la sociedad estas personas se adapten con mucha más calidad. Se evidencia que la mejor forma de generar inclusión de las personas con discapacidad es por medio del deporte adaptado; con el cual se fortalece su autoestima, relaciones interpersonales, este transforma vidas y se ve reflejado en las 7 historias de vida de los jóvenes que participaron en el estudio, quienes resaltan que se ha convertido en su fortaleza.

El análisis de estas historias de vida permite confirmar que la práctica del deporte adaptado contribuye a elevar la calidad de vida de estas personas y haciéndolos sujetos de derechos como lo indica la convención de las naciones unidas. El deporte inclusivo brinda la oportunidad de que jóvenes practiquen un deporte de acuerdo con su condición fortaleciendo su actividad física, donde se cultivan valores y actitudes, cabe mencionar que estas propicias interacciones sociales.

El deporte se convierte en espacio donde las personas con discapacidad logran pasar la página de una discapacidad, pasarla en el sentido de todo lo relacionado con esa fase de duelo por adquirir o nacer con una discapacidad, así mismo con el deporte las personas con discapacidad pueden escribir una nueva historia en su vida, una página cargada de esperanza y superación donde a través de sus vivencias logran ser un ejemplo de vida para toda la sociedad.

El deporte aporta al mejoramiento de la calidad de vida de los atletas sobre todo en su rehabilitación dado que muchos jóvenes con discapacidad se les dificulta acceder al sistema de salud o si acceden este se demora en autorizar servicios básicos como las terapias, entonces el deporte aporta considerablemente como un soporte para sus terapias. La mayoría de los entrevistados considera la discapacidad como algo que solo se encuentra en la mente de las personas, esto se evidencia en sus relatos, los pensamientos hacen soñar cosas que a veces se consideran inalcanzables pero la motivación es la fuente 
que alimenta estos sueños y los vuelve realidad.

Estas historias de vida pueden ser llevadas a diversos contextos con el fin de sensibilizar a la sociedad y que a través de estas historias se puedan aportar a que muchos jóvenes se motiven a salir adelante independientemente de su condición. En el departamento de Córdoba se cuenta con muchos jóvenes que practican deporte inclusivo, algunos han podido acceder a la educación superior gracias a su condición de deportista, sin embargo es necesario resaltar que se requieres más escenarios de participación para ellos de más apoyo gubernamental.

Se concluye que existen unas barreras, limitaciones, que padecen enfrentan las personas con discapacidad y son los impedimentos sociales, económicos, acceder a un empleo son los que limitan su integración. El departamento de Córdoba carece de espacios para incluir a la población con discapacidad y que solo en el deporte encuentran oportunidades para mostrar sus talentos y para convertirse en ejemplos de vida para todas las generaciones

\section{Referencias}

Alheit, P. \& Dausien, B. (2008). Procesos de formación y aprendizaje a lo largo de la vida. Revista de la Asociación de Sociología de la Educación, 1(1). 25 28.

Arraez, M. J. (2013). El deporte adaptado: historia, práctica y beneficios. Málaga: IAD.

Bandura, A. (1986). Social foundations of thought and action: A social cognitive theory. Englewood Cliffs, NJ: Prentice-Hall.

Bandura, A. (1999) Autoeficacia: como afrontamos los cambios de la sociedad. Chile, pág. 223-225

Blasco-Mira, M. \& Pérez-Turpin, J. (2007). Metodología de la investigación en Ciencias de la Actividad Física y el Deporte. Editorial Club Universitario

Cajamarca, C. (1994) Aprender a educarse, a ser y a obrar. Editora Géminis Ltda,

Díaz-Better, H. R. \& Eusse-López, C. (2021). Uso del deporte Boccia como herramienta de inclusión en la clase de educación física: un estudio de mapeo sistemático. VIREF Revista De Educación Física, 10(1), 96-107.
Gómez-Bohórquez, W. (2019). Historias de Vida de Profesionales con Discapacidad en el Contexto de Educación Física, Deporte y Recreación. Universidad Pedagógica Nacional.

Gómez-Rodríguez, M. (2017). Inclusión Laboral de Docentes con Discapacidad Visual en el Campo Educativo. Universidad Pedagógica Nacional.

Hernández-Sampieri, R., Fernández-Collado, C. \& BaptistaLucio, N. (2014). Metodología de la Investigación. 6ta edición. McGraw-Hill / Interamericana Editores, S.A.

Kearney, M., Orrell, R., Fahey, M., Brassington, R. \& Pandolfo, M. (2016). Pharmacological treatments for Friedreich ataxia. Cochrane Database of Systematic Reviews, 8. Art. No.: CD007791. DOI: 10.1002/14651858.CD007791.pub4

Montes-Delgado, R., Palos-Toscano, U. \& Avalos-Latorre, M. (2016). Influencia de la Práctica del Deporte Adaptado sobre la Calidad de Vida: Un Estudio Cualitativo. EmásF, Revista Digital de Educación Física. (8)43. http://emasf.webcindario.com

Organización Mundial de la Salud (OMS) (2001). Clasificación Internacional del Funcionamiento, de la Discapacidad y de la Salud. Ministerio de Trabajo y Asuntos Sociales. Secretaría de Estado de Servicios Sociales, Familias y Discapacidad. Instituto de Mayores y Servicios Sociales (IMSERSO).

Pérez, T., Blasco, Y., González, L., García, H., Soto, R. \& Coterón, J. (2013). Paraciclismo: estudio sobre los procesos de integración a nivel internacional. Apuntes: Educación Física y Deportes, 111, 79-86.

Salazar-Arbués, J. \& Escós-Julián, A. (2020). El Deporte inclusivo en las aulas. Universidad de Zaragoza.

Taylor, S., Bogdan, R. \& DeVaiult, M. (2016). Introduction to qualitative research methods: A guide and resource $\left(3^{\mathrm{a}}\right.$ ed.) 